\title{
Genital Warts and Treatment Options:
}

\author{
Clinical Audit \\ Helen Patrick ${ }^{1}$, A.A.I.N Jayasekara ${ }^{1,2}$, I.D.C Wijerathna ${ }^{2}$, Colin Fitzpatrick ${ }^{1}$, \\ Fionnuala Finnerty ${ }^{1}$, Rebecca Murdock ${ }^{1}$, Daniel Richardson ${ }^{1,3}$ \\ 1. Brighton \& Sussex University NHS Trust, Brighton \\ 2. University of Peradeniya, Sri Lanka \\ 3. Brighton \& Sussex Medical School, Brighton
}

\section{Background}

Genital warts are a common condition that present in sexual health clinics. There are different treatment options available within BASHH guidelines depending on the size and location of warts. Home treatment reduces follow up clinic attendance.

\section{Methods}

We randomly selected 30 patients attending our integrated sexual health service with new genital warts and audited their management against local guidance that home treatment with imiquimod should be used first line for 4 weeks, unless contraindicated. ${ }^{1}$

\section{Results}

- Of the 30 patients, 18 were male and 12 were female.

14/30 were prescribed imiquimod only.

-7 patients had cryotherapy only

- 9 were also given cryotherapy before imiquimod.

- 9/16 who received cryotherapy requested this treatment. 6/16 had documented reasons why it was deemed appropriate to have ${ }_{20}$ cryotherapy (unable to apply cream themselves, site of lesion).

- Interestingly, all 9/9 (100\%) who had received combination treatment reported clinical resolution within 4 weeks.

6/7 (86\%) who had cryotherapy only clinically resolved after 3

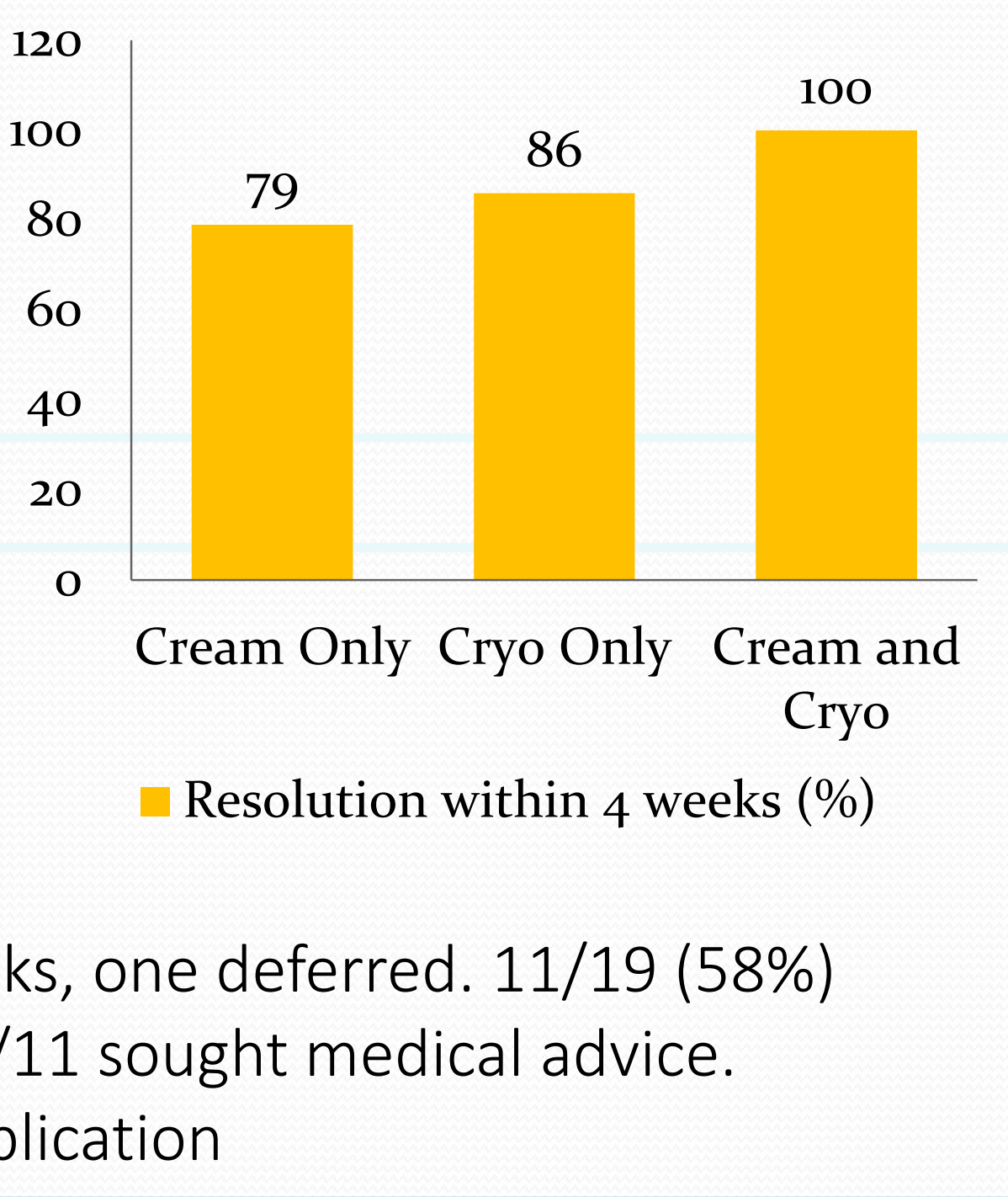
consecutive applications.

11/14 (79\%) treated with imiquimod only resolved within 4 weeks, one deferred. 11/19 (58\%) treated with imiquimod experienced side effects and 5 patients $5 / 11$ sought medical advice.

-There were no reported complications following cryotherapy application

\section{Conclusion}

Despite imiquimod being the recommended first line initial treatment for genital warts in our service, some patients received cryotherapy treatment in isolation or a combination of imiquimod and cryotherapy. The patients receiving cryotherapy were likely to have requested this treatment and had fewer side effects. All patients who received imiquimod and cryotherapy had resolution of genital warts in four weeks.

\section{References}

1. UK National Guidelines on the Management of Anogenital Warts 2015. Clinical Effectiveness Group, British Association Sexual Health and HIV (BASHH)

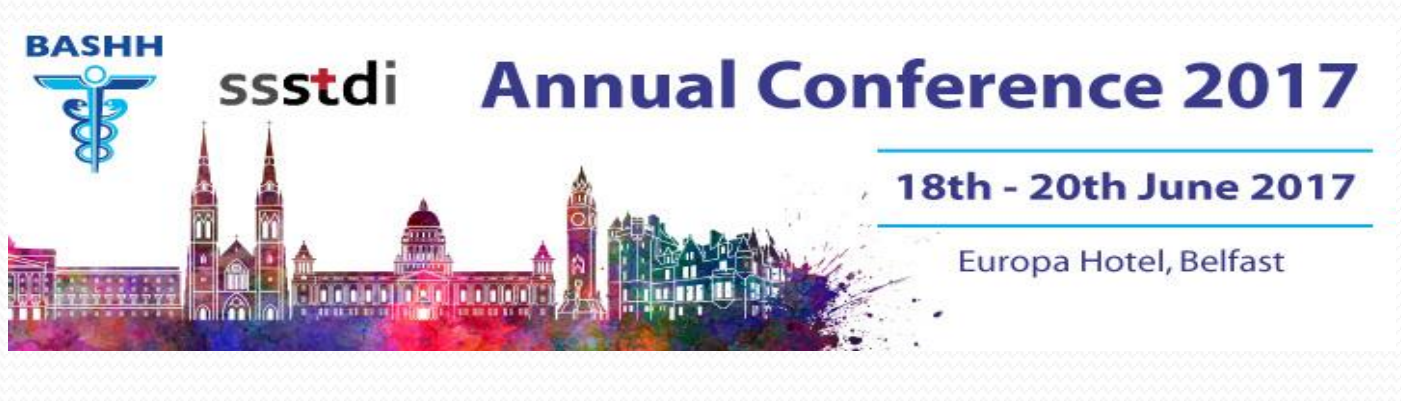

